\title{
OBSERVATIONS ON OTITIS MEDIA AND MASTOIDITIS IN INFANCY
}

BY

\author{
EDWARD C. ROSS COUPER, M.D., F.R.F.P.S., D.P.H.
}

Deputy Medical Superintendent, Walton Hospital, Liverpool

There have recently been articles and correspondence in the medical journals regarding the relationship between mastoiditis and the diarrhoea and vomiting syndrome in infancy. It is thought that some observations, made mainly from a detailed investigation of a series of fifty infants suffering from this infection conducted a few years ago in Alder Hey Childrens' Hospital, on this important problem might be of interest. All the cases were thoroughly investigated to exclude other infections.

At the outset it must be emphasized that diarrhoea and vomiting are the two commonest signs of disease in infancy and that inflammation of the middle ear and mastoid antrum is only one of the many sources of parenteral infection which may give rise to them. In the recent articles and letters stress has been laid only on mastoiditis and it should be mentioned that otitis media alone may give rise to almost as serious an illness in an infant as the case which has progressed to mastoiditis. There is no doubt that these infections can cause serious illness, and it would seem that in some cases otitis media alone can be sufficient to produce a fatal issue.

Anatomy

There are one or two anatomical points which it is useful to keep in mind. The Eustachian tube is relatively wider and more horizontally placed than in the adult (Leathart, 1943). The infantile tympanic membrane lies at a much more acute angle and may be almost horizontal (Lyman, 1927a). This accounts to some extent for the difficulty in examining the tympanic membrane, the object surveyed appearing oval rather than circular. At birth a soft mesodermal embryonic tissue, which presents a myxomatous appearance on section, fills the antrum, attic and upper portion of the middle ear and facilitates the spread of infection (Lyman, 1927a; Marriott, 1925a and b). It is said that this embryonic tissue also lines the inner aspect of the tympanic membrane rendering it thicker and explaining the infrequency of spontaneous perforation in the infant (Le Mée, 1936). Lyman (1927a) suggested that in cases in which the gastro-intestinal symptoms improve with myringotomy alone, the infection is confined to the lower part of the middle ear cavity and that when infection has spread to the upper part with its embryonic tissue myringotomy does not provide sufficient drainage. Inflammation and swelling of this tissue hampers adequate drainage. Fig. 1, which is a microphotograph of a normal infantile mastoid, shows this tissue filling one of the air cells.

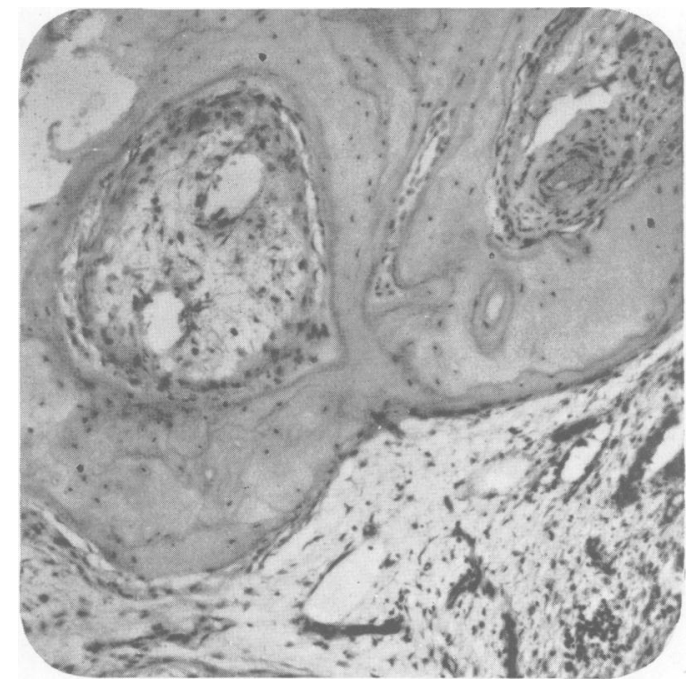

FIG. 1.

\section{Bacteriology and pathology}

Swabs were taken from all cases with otorrhoea and after myringotomy, and from the nasopharynx of all infants. Cultures from these swabs invariably produced a mixed growth and in nearly all staphylococci predominated, particularly in the ear swabs. Swabs were taken at operation and in the postmortem room with similar results. The results of this part of the investigation lead to no satisfactory conclusion. Blood cultures proved negative with one exception, in which a haemolytic staphylococcus was obtained. In 64 per cent. of the families a history of recent respiratory infection was found, e.g. 'colds,' sore throat, bronchitis, pneumonia and influenza, and a recent leading article in the British Medical Journal (1943) has raised the question of staphylococcal pneumonia. Recently several infants have been observed suffering from staphylococcal pneumonia and it is possible that the staphylococcus has some pathogenic significance in otitis 
media and mastoiditis. Certain staphylococci are known to elaborate an enterotoxin but what part, if any, this plays in producing the gastrointestinal symptoms is pure conjecture. Many attempts have been made to establish a satisfactory bacteriology

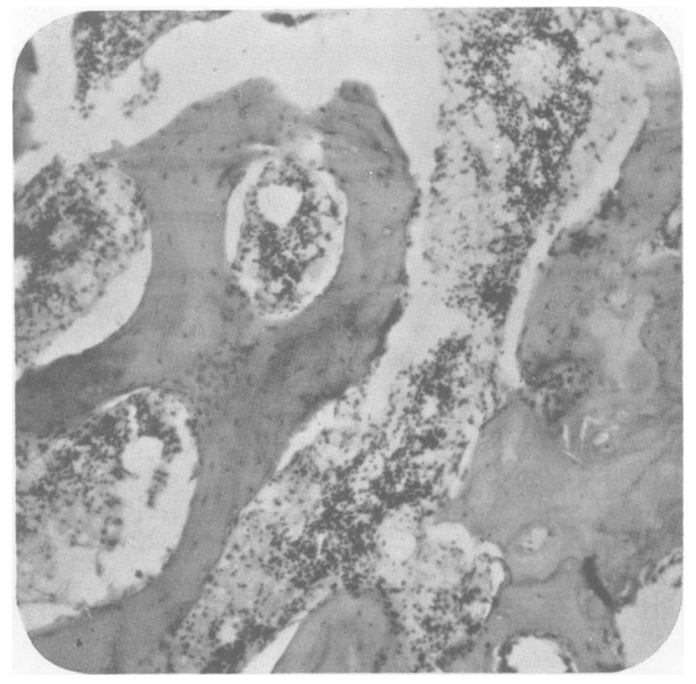

FIG. 2.

without convincing result, the commonest organisms found being staphylococci and streptococci. Alden (1927) found haemolytic streptococci in the 'latent' type of mastoiditis whilst Spahr (1929) found staphylococci the commonest organisms in cultures from the middle ear. Marriott (1925a) stresses the importance of haemolytic streptococcal infection of the mastoid antra in his cases; Smith (1924) and Holsclaw et alia (1930), also found them the commonest organisms. Fig. 2 shows a section of an infected mastoid process in which many pus cells can be seen in the embryonic tissue occupying the

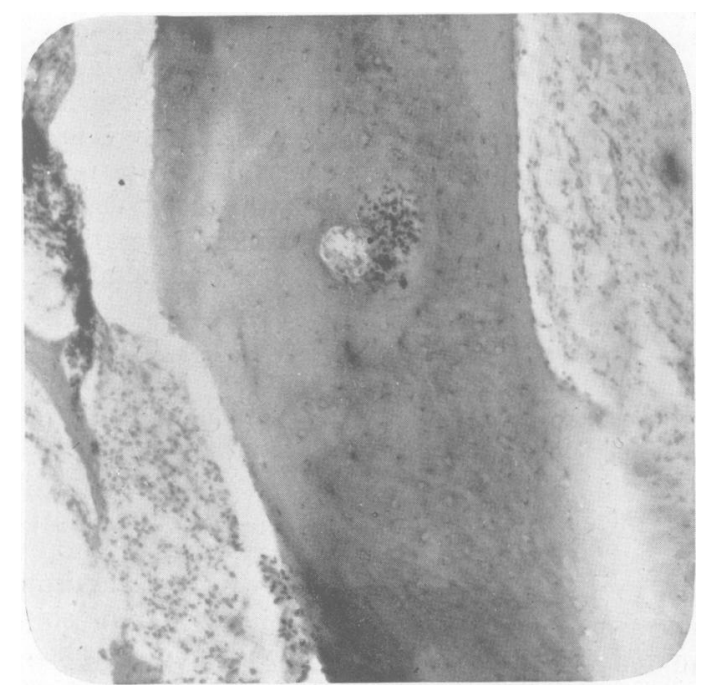

FIG. 3. air cells. In fig. 3 the section appears to cut the tip of an air cell but pus cells can be seen in the bony wall.

\section{Etiology}

It seems likely that the majority of these infections result from upper respiratory infection derived from those in attendance. As Leathart (1941) points out the anatomical peculiarities of the Eustachian tube lend themselves to infected mucus and discharges making their way into the middle ear and setting up an otitis followed in certain cases by mastoiditis. It is possible that infected milk may have a similar effect although no fluid recognizable as milk has been noted in the middle ear at post-mortem examination. Once the infection has begun, the embryonic tissue already mentioned assists the spread of the infection. Dean (1927a and b) stressed the importance of paranasal sinus disease in producing a clinical picture of gastro-enteritis and suggested that aural infection and paranasal sinus disease may coexist-a pansinusitis. If this is so, it may explain failure of treatment directed against aural infection alone. As in all infections of infancy, bottle-fed infants are more likely to develop aural infection. In the series investigated the great majority had less than three months of breast feeding. Only two were entirely breast fed and only three had more than three months. The subsequent bottle feeding was fairly satisfactory but the supply of vitamins was most unsatisfactory. Seventeen cases had no vitamins at all.

\section{Incidence}

Aural infection can occur in any infant, the youngest case noted being only five days old. In 1324 infants under one year (McConkey and Couper, 1938) it was found that 195 (14 per cent.) had suffered from aural infection. Of the 195 cases, 116 were associated with other conditions, e.g. respiratory infections, athrepsia, nasopharyngitis. In seventytwo cases aural infection was the only detectable disease and seven cases presented the ordinary classical signs of acute mastoiditis. Cooper found that otitis media was by far the commonest parenteral infection, occurring in 26 per cent. of his cases, while Ebbs (1937) found otitis media in 61 per cent. of infants dying in the first year of life. The view (Leathart, 1941), based on calculation from a series of 72 post-mortems among whom 59.6 per cent. were found to have mastoiditis, that, of 30,000 infants dying annually from gastro-enteritis 18,000 suffer from mastoiditis and that 14,400 of these could be saved by operative interference, is not in keeping with the experience of the present writer.

\section{Signs}

(1) General. The commonest signs in order of frequency are diarrhoea, vomiting, loss of weight and restlessness. If the diarrhoea and vomiting are severe then the infant is in danger of becoming dehydrated and toxic. The onset of dehydration is an occurrence of the greatest importance as it has a 
bearing on the treatment to be described later. In fact, cases of aural infection can conveniently be divided into two groups- those which are dehydrated and those which are not. It is interesting that the incidence of otorrhoea is higher in the non-dehydrated cases than in the dehydrated cases. In 39 non-dehydrated cases (McConkey and Couper, 1938) 33.3 per cent. had otorrhoea on admission to hospital while in 33 dehydrated cases only 9 per cent. had otorrhoea. This suggests that adequate drainage has a bearing on the development of dehydration and toxicity. When efficient drainage from the middle ear in cases of otitis media can be maintained by myringotomy, repeated if necessary, dehydration does not supervene. When drainage is difficult to maintain and dehydration occurs probably a proportion of cases have developed mastoiditis. On the other hand, in cases in which copious discharge has persisted for four weeks or longer with the child in good condition, mastoiditis has also probably developed. The temperature chart has not been found to be of much assistance but on the whole fever is higher in the dehydrated toxic cases than in those not so affected.

(2) Local. Examination of the tympanic membrane will frequently lead to the detection of this source of parenteral infection. The appearances of the membrane vary greatly. In some cases it is acutely inflamed, red and bulging or only mildly injected. In some cases it merely lacks the normal lustre, while in others it is apparently normal. The highest percentage of obviously pathological drums is found in the dehydrated toxic patients. Sometimes in these cases an apparently normal drum has pus behind it and the infant may also have mastoiditis. Sometimes myringotomy may be performed without apparent result, but when dehydration is overcome discharge commences. Alden (1927) explained the absence of signs in the tympanic membrane by the fact that the infant's Eustachian tube is shorter and wider than the adult's and drainage can take place more easily by this route. In other cases small unnoticed amounts of discharge may be detected by aural examination. Some parents seem to regard a little aural discharge as a normal occurrence and the dangers of this theory should be thoroughly explained.

There are two types of infantile mastoiditis. In the 'latent' type none of the ordinary signs is present. There is no swelling, redness, or oedema over the mastoid process, which renders the diagnosis extremely difficult. Leathart (1943) sets great store by enlargement of glands in the posterior triangle of the neck. Unfortunately there are other causes of such enlargement and it has not been possible to confirm the value of this sign. The second type is the ordinary classical mastoiditis which presents the usual signs of redness, oedema, and forward displacement of the pinna. The latter type is unfortunately much rarer than the former and the infant never becomes dehydrated. Having regard to the frequent bilateral occurrence of aural infection in infancy the possibility of a 'latent' mastoiditis on the other side should be kept in mind. One such case has been observed and the 'latent' mastoiditis ultimately lead to a fatal result.

\section{Diagnosis}

Examination of the tympanic membrane, without which no examination of an infant is complete, is of the greatest importance and may lead to the real cause of gastro-intestinal signs being discovered. Provided an electric auroscope with a small speculum is used it is always possible to examine the drum except in very small infants such as premature infants. The difficulty lies in the interpretation of the findings and this is a matter of experience. The most difficult drums, fortunately not many, are those which appear normal, and these may quite reasonably be missed. The more obvious degrees of inflammation are quite easily detected. Both tympanic membranes should always be inspected and if one ear is already discharging at the time of the examination, the ear without discharge should obviously be examined first in order not to convey infection to a possibly healthy ear.

The diagnosis of the classical type of mastoiditis is fairly easy, but when attention is directed to the ear by changes in the tympanic membrane, the diagnosis of the 'latent' type presents a very difficult problem. The diagnosis can best be made by considering the general condition of the infant, particularly the progress of its weight curve, the severity and duration of the illness, the duration of aural discharge, the presence of changes in the tympanic membrane and response to treatment. A sudden drop in weight is suggestive of parenteral infection of this type.

\section{Treatment}

It is not proposed to deal with the treatment of dehydration and only the treatment of aural infection will be considered. On the whole such treatment should be as conservative as possible.

(1) LOCAL MEDiCATION. For acutely inflamed drums before and after discharge has commenced, instillation of ' glycerin and carbolic' drops is useful. Later as the acute phase passes spirit drops may be employed with benefit.

(2) Myringotomy. This minor operation is easy to perform and in some cases can be almost a life saving measure. No anaesthetic is necessary and the operation is best performed through the speculum of an electric auroscope. The anatomy previously described should be kept in mind. The difficulty lies in recognizing when to perform the operation. This is a matter of correct interpretation of the signs seen in the drum. When the drum is acutely inflamed and bulging, the decision is easily made but many much less pathological drums have been opened and pus released. A single myringotomy is sometimes sufficient and the general condition and gastro-intestinal symptoms improve. After a successful myringotomy the drum should be 
inspected daily because in the infant the opening seems to be peculiarly prone to seal over rapidly, thus preventing discharge of pus. In some cases maintenance of drainage is difficult and several myringotomies may be necessary before the infection is overcome. Note of aural discharge should be made on the weight chart. If this is done it will be noticed that if discharge ceases before the infection is overcome the infant either fails to gain or actually loses weight. On the other hand, if discharge ceases and the infant is gaining weight satisfactorily it is probable that infection has been overcome. In the former case a further myringotomy may be necessary perhaps in the same drum or the other drum. As in all infantile diseases the weight curve is an extremely useful guide to the success or otherwise of treatment. One explanation has been offered for the unfruitful myringotomy, and spontaneous drainage via the Eustachian tube is another suggested explanation (Alden, 1927).

(3) SURgical DRAinage OF THE MASTOID ANTRUM. In the classical case the decision is easy and operation is the treatment of choice. In the 'latent' type the decision to operate is an extremely difficult one. The factor which should influence this decision most is the presence or absence of dehydration. In my opinion, operation, if indicated, may be risked in the mildly dehydrated infant but in the severely dehydrated, toxic child operation should never be undertaken. If, however, severe dehydration can be overcome by the usual methods and the infant's general condition improved then operation may be carried out with much greater safety if indicated. The type of case in which good operative results may be obtained is that in which the aural infection has become more chronic with three or four weeks otorrhoea and the infant's general condition is good. It is felt that this decision is one which should remain in the hands of the paediatrician as the most dire result of aural infection, namely the onset of dehydration and toxicity, is a paediatric problem and the paediatrician is best able to say when the infant is fit for operation.

The published results are interesting.

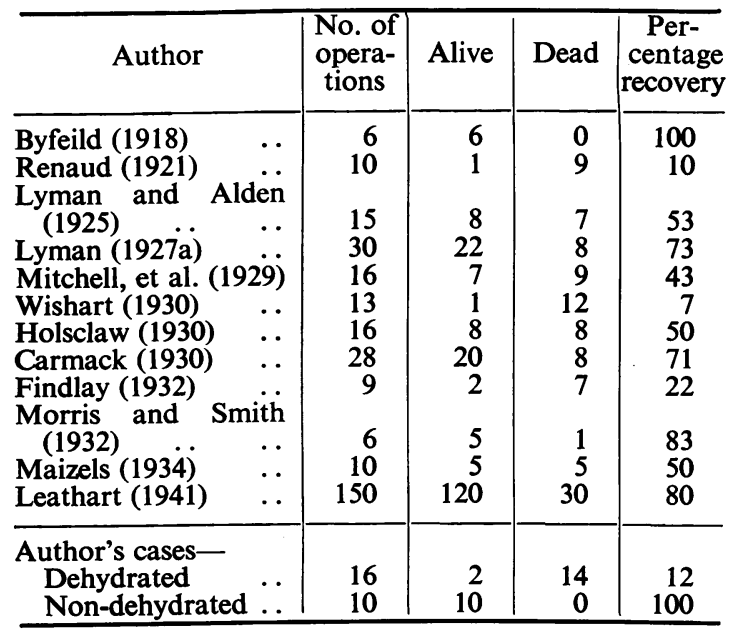

The majority show a recovery rate of over 50 per cent. My cases are divided into two groups, and it can easily be seen in which group the best results are obtained. Carmack (1930) is of the opinion that operation should never be carried out in the acute phase of the illness, and it was found (McConkey and Couper, 1938) that 80 per cent. of dehydrated cases which were operated upon died and that 86 per cent. of such cases treated conservatively died. Surgical drainage of the mastoid antrum in severely ill infants without some local indication, ' a shot in the dark,' is unsound. Many such cases have come to the post-mortem room when it has been demonstrated quite clearly that operation would have been of no avail.

(4) SulphONAMIDES. More recently treatment of acute otitis media with sulphadiazine has been attempted. At present the series is too small to allow of conclusions being drawn, but it would seem that, in cases with an acutely inflamed drum and no discharge, sulphadiazine in full doses causes rapid improvement. It is advisable to continue the drug, which is very well tolerated, for about a week, otherwise relapses may occur. On the other hand, once otorrhoea has commenced the exhibition of sulphadiazine appears to exert no beneficial effect. The explanation of this is not clear. Possibly after otorrhoea has begun the infection becomes a mixed one and therefore less amenable to the drug.

\section{Prophylaxis}

(1) Infection. Persons suffering from colds and sore throats should on no account come into contact with infants. Attendants, nurses and doctors should invariably wear masks. Mothers seem reluctant to wear masks even when the reason has been explained. Nevertheless facilities should be available, e.g. a supply of masks at clinics, so that mothers can wear masks even at home on the slightest suspicion of respiratory infection. Demonstrative fondling and kissing of infants by relatives and friends cannot be too strongly deprecated.

(2) Position. For reasons already given, very weakly and ill infants should as far as possible be nursed in a position approximating to Fowler's position.

(3) Feeding. Infants should always be fed in a semi-upright position and when bottle fed should if possible be taken out of the cot to be fed. Bottles should never be left in the cot beside a recumbent infant.

(4) Isolation. In hospitals and institutions infants should always be nursed according to a strict barrier nursing technique with gowns and masks. This should be a routine and not merely introduced when some epidemic occurs. The ideal system is of course cubicle wards, but it is possible to carry out the technique efficiently in an open ward provided overcrowding is avoided. Difficulty arises when the mothers have to come into contact with their infants, and they ought to be instructed in carrying out the technique. 


\section{Post-mortem examinations}

Many autopsies have been performed and the following are the noteworthy features.

(1) The almost invariable bilateral occurrence of infection of the mastoid antra.

(2) The absence of intracranial complications, such as sinus thrombosis and intracranial abscess found in older patients.

(3) The absence of intestinal lesions sufficiently gross to account for the extreme illness of these infants.

(4) In certain cases the impression was gained of a severe toxaemic or septicaemic state, although the latter view is not supported by the results of blood culture.

\section{Summary}

Eclampsia has been described as the "disease of theories.' Aural infection in infancy might well be described as the 'disease of problems.'

(1) Can aural infection be an adequate explanation of gastro-intestinal signs ? In my opinion a certain proportion of such cases can be so explained although this view has not gained widespread acceptance.

(2) The bacteriological investigations so far published are unsatisfactory. A suggestion has been made regarding the pathogenicity of the staphylococcus and perhaps further investigation would be fruitful.

(3) The difficulty of diagnosis in 'latent' mastoitditis has been described. How can the diagnosis be made in the absence of satisfactory local signs?

(4) In hospital the problem of infection can be largely overcome, but outside it is a different matter and is really a sociological problem. Overcrowding and bad housing must exert a great influence on the spread of infection.

(5) Breast feeding should be encouraged in every possible way. Some mothers seem disinclined to breast feed their infants, especially in the present difficult circumstances.

Thanks are due to Dr. W. E. Crosbie, Medical Superintendent, Alder Hey Childrens' Hospital, Liverpool, for permission to publish these results.

\section{REFERENCES}

Alden, A. M. (1927). Arch. Otol., 5, 39.

Brit. med. J., 1943, 2, 783.

Byfeild, A. H. (1918). J. Amer. med. Ass., 71, 511.

Carmack, J. W. (1930). Ann. Otol. etc., St. Louis, 39, 75 .

Cooper, E. D. (1937). $\quad$ Arch. Dis. Childh., 12, 339.

Dean, L. W. (1927a). Ann. Otol. etc., St. Louis, 36, 933. (1927b). Arch. Otol., 6, 201.

Ebbs, J. H. (1937). Proc. roy. Soc. Med., 30, 49.

Findlay, L. (1932). Arch. Dis. Childh., 7, 307.

Holsclaw, F. M., Boehm, C. A., and Bierman, J. M. (1930). Amer. J. Dis. Child., 39, 747.

Leathart, P. W. (1941). J. Laryng., 41, 320. (1943). Brit. med. J., 2, 168.

Le Mée, J. M. (1936). Bull. Soc. Pédiat. Paris, 34, 33.

Lyman, H. W. and Alden, A. M. (1925). Laryngoscope $35,586$.

Lyman, H. W. (1927a). Arch. Otol., 6, 526.

(1927b). Ann. Otol. etc., St. Louis, 36, 903.

Maizels, M. (1934). Lancet, 1, 1329.

Marriott, McK. (1925a). Amer. J. Dis. Child., 30, 577. (1925b). Laryngoscope, 35, 592.

Mitchell, A. G., McCarthy, M. F., Leichliter, J. W., and Seinsheimer, F. (1929). J. Amer. med. Ass., 92, 970.

Morris, M., and Smith, W. B. (1932). Amer. J. Dis. Child., 44, 964.

McConkey, G., and Couper, E. C. R. (1938). Arch. Dis. Childh., 13, 137.

Renaud, M. (1921). Bull. Soc. méd. Hôp. Paris, 45, 1326.

Smith, D. T. (1924). Amer. Jour. Dis. Child., 28, 1.

Spahr, M. B. (1929). Ibid., 37, 541.

Wishart, D. E. S. (1930). J. Amer. med. Ass., 95, 1084. 